Advances in Radio Science (2004) 2: 163-167

(C) Copernicus GmbH 2004

\title{
On UWB beamforming
}

\section{T. Kaiser}

Duisburg-Essen University, Faculty of Engineering, Department of Communication Systems, Bismarckstraße 81, 47048 Duisburg, Germany

\begin{abstract}
Ultra-Wideband (UWB) communication systems and Multi-Input-Multi-Output (MIMO) techniques rank among the few emerging key technologies in wireless communications. For that reason the marriage of these two complementary approaches should deserve attention. Apparently, the extremely large ultra-wide bandwidth creates rich multipath diversity which calls, at a first glance, additional antenna elements into question. However, another point of view is as follows. The attenuation by solid materials usually increases with increasing frequency; e.g. frequencies above, say, $10 \mathrm{GHz}$ are considered to be blocked by walls etc. Since UWB can occupy more than $7 \mathrm{GHz}$ of bandwidth (according to FCC regularisation) the performance of a communication link can be physically extended only by adding spatial information, i.e. multiple antennas, even if such extension may play a minor role. From this point of view UWB\& MIMO presents an upper physical bound for indoor communications and is therefore at least worth to be investigated. In order to see the forest for the trees, we will focus in this limited contribution on beamforming among all alternative MIMO techniques (like space time coding or spatial multiplexing).
\end{abstract}

\section{Introduction}

In order to avoid strong interference of conventional narrowband transmission systems by ultra-wideband signals, the power spectral density of UWB systems is fairly limited, which leads - despite the enormous bandwidth - to a rather restricted coverage. This weakness is opposite to one of the main strengths of MIMO techniques; they are able to increase the range. Such a reversal indicates the potential of a marriage of these two complementary techniques.

Numerous other benefits can be envisaged. For example, the capability of MIMO systems to spatially distinguish among wavefronts impinging from different directions not

Correspondence to: $\mathrm{T}$. Kaiser

(thomas.kaiser@uni-duisburg.de) only facilitates the equalizer design of UWB systems by reduction of delay spread, but rather enables a further increase of data rate - Gbit/s over air becomes feasible. Moreover, by MIMO techniques, narrowband and broadband interferers can be spatially suppressed so that the number of concurrent users might be significantly increased. Last but not least a reduction of electromagnetic radiation can be expected from UWB \& MIMO, which in turn may also save battery life.

However, a large number of different challenges do resist. For example, digital beamforming seems to be prevented due to the extremely high sampling rate. In contrast, analog beamforming requires adjustable true time delays, such delays exhibit noticeable tolerance and therefore less precision. Besides these technology barriers, numerous challenges have to be overcome in fundamental processing of UWB signals by MIMO techniques.

\section{An analogy}

Without reinventing the wheel, some ideas can be borrowed from speech or also from radar processing. The following table shows the similarities and differences of broadband beamforming for speech processing and UWB communication systems.

Apparently, the relative bandwidth is of the same order and the delay spread differ by a factor $10^{6}$, which is caused by the ratio between speed of propagation of light and sonic waves. Furthermore, the environment is the same as well as the propagation phenomena, e.g. multipath or broadband reflection by obstacles. Because fading is not so severe in UWB communications (Cramer et al., 1999; Lee et al., 2000) it is reasonable to assume some degree of signal correlation among the antenna elements which results in regular shaped wavefronts. The concrete type of the wavefront, i.e. spherical or planar, depends on the ratio between antenna aperture and transmitter-receiver distance. In order to guarantee a useful transmission, the signal to noise ratio at the receiver has to 
Table 1. An analogy between speech processing and UWB transmission.

\begin{tabular}{ccc}
\hline Property & Speech Processing & UWB transmission \\
\hline bandwidth (rel.) & $\approx 10 \mathrm{kHz}(180 \%)$ & $\approx 10 \mathrm{GHz}(110 \%)$ \\
delay spread & $\approx 100 \mathrm{~ms}$ & $\approx 100 \mathrm{~ns}$ \\
environment & indoor, non-stationary & indoor, non-stationary \\
challenges & highly reverberant & multipath propagation \\
wavefront shape & spherical or planar & spherical or planar \\
typ. SNR at receiver & moderately positive $(6-12 \mathrm{~dB})$ & moderately positive $(6-12 \mathrm{~dB})$ \\
receiver type & waveform estimation & detection (matched filter!) \\
type of interferers & broadband & broadband and narrowband \\
type of noise & correlated (e.g. ventilator) & uncorrelated \\
other issues & signal duration unknown & signal duration known \\
\hline
\end{tabular}

be moderately positive, say a $6-12 \mathrm{~dB}$. This can be achieved either in case of limited range or by spreading techniques.

All these mentioned aspects show the similarities between speech and UWB spatial processing. The main differences result from the fact that the UWB receiver knows the principal waveform whereas in speech processing not only the waveform is completely unknown but also when speech begins and when it ends. From this point of view, UWB seems to be easier to deal with because the transmit signal can be designed adequately. For example, in order to estimate the direction-of-arrival $\theta$ of an impinging wavefront (see Fig. 1), the transmit signal can be repeated periodically so that at the receiver the sampling rate can be distinctly reduced by almost arbitrary low undersampling techniques. Moreover, for UWB transmission a matched filter (MF) can be principally deployed that further boosts up the SNR. However, this approach tends to be a bit overrated, since in dense UWB multipath environments numerous reflections occur and each individual reflection will modify the transmitted impulse shape simply because of the non-flat frequency response of the reflective material. One last major difference concerns the kind of noise and interferers. While in speech processing broadband interferers dominate and the noise is most often correlated (e.g. ventilator), in UWB transmission the interferers can be broadband as well as narrowband and the noise is mainly of thermal type and hence uncorrelated.

In conclusion, because of its affinity, some concepts - like broadband beamforming - can be borrowed from speech processing.

\section{Broadband beamforming}

As mentioned previously we will focus on beamforming of UWB signals. The following figure illustrates a conventional filter and sum beamformer.

Basically, each filter can be substituted by a delay in order to compensate for the different signal travel time among the antenna elements and to coherently summing up all branches. This so-called array gain enhances the SNR by a factor of $N$ (or $10 \log _{10}(N)$ on a decibel scale) under perfect conditions.

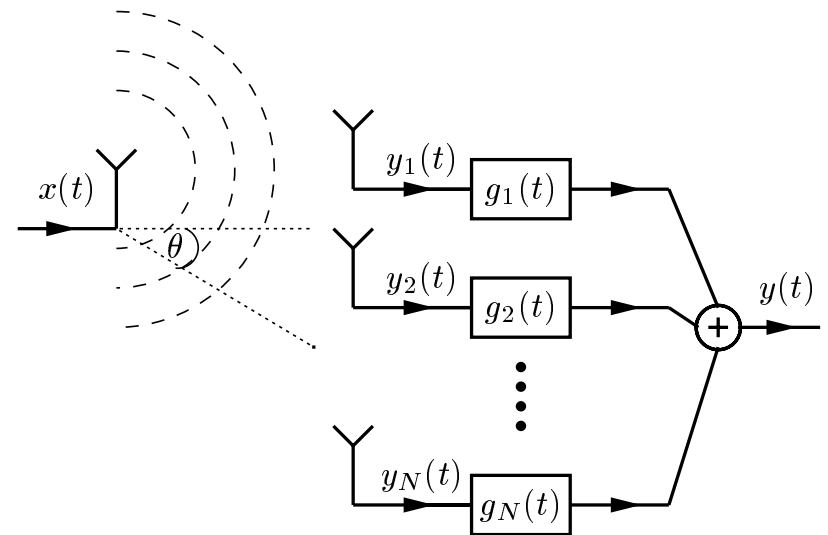

Fig. 1. A filter and sum broadband beamformer.

However, interferers cannot be suppressed only by delays. For that reason, the use of filters becomes justified. Figure 2 shows the beampattern defined as

$B\left(\theta, \theta_{0}\right)=\max _{t}\left|y\left(t, \theta, \theta_{0}\right)\right|^{2}$

for a family of sinusoidal signals

$x(t)=\sin (2 \pi f t), \quad f=3.1 \mathrm{GHz} \ldots 10.6 \mathrm{GHz}$

and $N=8$ antennas, a steering direction of $\theta_{0}=0^{\circ}$, an antenna spacing $d=\lambda_{c} / 2$, where $\lambda_{c}=c_{0} / f_{c}, c_{0}$ is speed of light and $f_{c}=6.85 \mathrm{GHz}$ is the center frequency according to $\mathrm{FCC}^{1}$-regularisation.

Observe that while the location of the mainlobe is frequency-independent, the locations of the sidelobes and the nulls are frequency-dependent. This means that broadband interferers cannot be completely suppressed by a simple delay and sum beamformer. Filters represent a remedy and allow an almost arbitrary high interferer cancellation just by adequately increase of the filter orders. In principle, conventional beamformer techniques (e.g. the minimum variance

\footnotetext{
${ }^{1}$ Federal Commission for Communications
} 


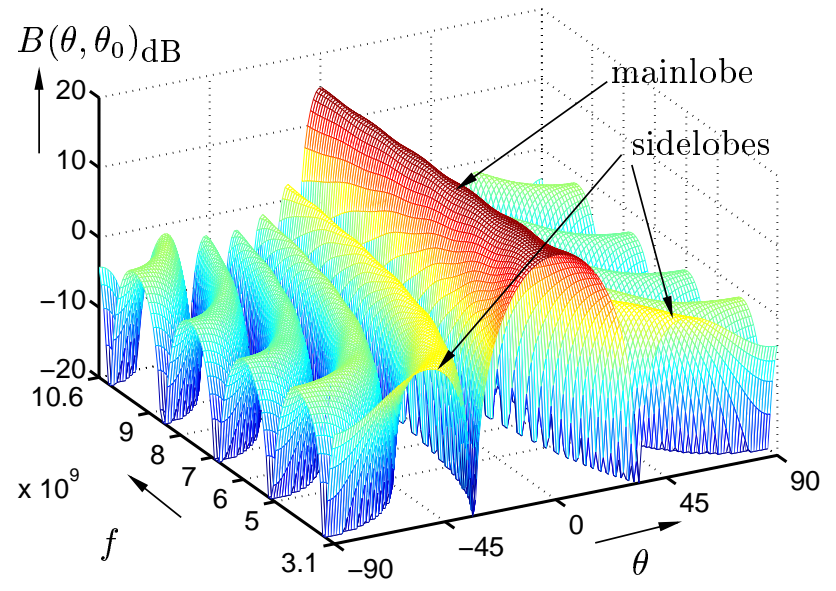

Fig. 2. Beampattern of a delay and sum broadband beamformer.

distortionless beamformer (MVDR)) can be deployed in order to design the filter and sum beamformer simply by calculating the weight vector for a set of suitable frequencies and transforming the resulting weight matrix into time domain.

UWB measurements have shown, at least under lineof-sight (LOS) conditions, the propagation of individual echoes arriving at the receiver in so-called clusters (see for example the descriptive videos at Whyless, www.whyless.org/public/wp5.htm). Due to their ultrashort duration, these echoes are resolvable in time so that they also become resolvable in angle; hence, direction-of-arrival estimation can be principally performed. Moreover, the recorded signals seem to be delayed replicas of each other so that beamforming becomes feasible. Even for a spacing of a few ten centimeters, the relevant echoes seem to be stongly correlated. In addition, a small spacing (e.g. $4 \mathrm{~cm}$ ) does not cause serious coupling (Sibille and Bories, 2003).

(Ultra)-broadband beamforming offers an additional benefit opposite to narrowband beamforming. Narrowband beamforming suffers from so-called grating lobes. They occur for antenna spacings $d$ larger than $\lambda_{c} / 2$ and they lead to an ambiguity of the direction-of-arrival. For that reason, common narrowband antenna elements show a spacing of half the carrier wavelength or even smaller than this. Taking into account that the mainlobe width depends not only on the number of antennas but also on the spacing, narrowband beams can only be further narrowed by increasing the number of antennas. In turn, in (ultra)-broadband processing also the spacing can be increased further without ambiguity. In order to illustrate this fact, the following figures (Figs. 3-6) show the squared magnitude of the beamformer output and the beampattern for an antenna spacing $d=\lambda_{c}$ in case of two antennas, lower $f_{l}=3.1 \mathrm{GHz}$ and upper cut-off frequencies $f_{u}=10.6 \mathrm{GHz}\left(\rightarrow f_{c}=6.85 \mathrm{GHz}\right)$, and an input signal $x(t)=\mathrm{e}^{-\mathrm{j} 2 \pi f_{c} t}$ (narrowband) or $x(t)=$ $\mathrm{e}^{-2 \pi\left(\left(f_{u}-f_{l}\right) t\right)^{2}} \mathrm{e}^{-\mathrm{j} 2 \pi f_{c} t}$ (broadband).

It can be seen that the narrowband beamformer will suffer from the mentioned ambiguity since it cannot distinguish

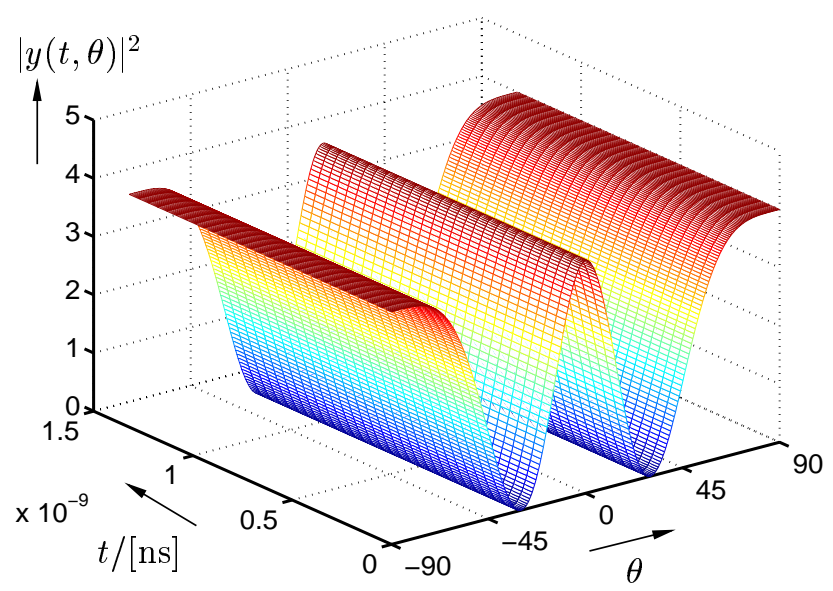

Fig. 3. Squared magnitude of narrowband beamformer output for $d=\lambda_{c}, N=2$

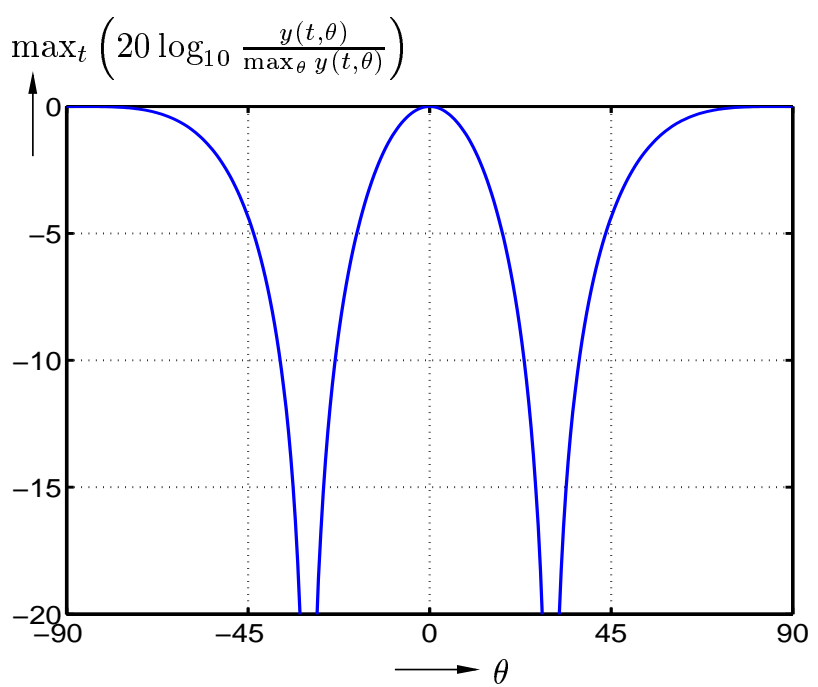

Fig. 4. Narrowband normalized beampattern for $d=\lambda_{c}, N=2$ in logarithmic scale.

among the three possible direction-of-arrivals $-90^{0}, 0^{0}$ and $+90^{\circ}$. In contrast, the broadband beamformer can clearly resolve the $0^{0}$-DoA, because of no ringing of the received impulse-shaped wavefront.

It is also of interest to study the effects caused by increasing the spacing and the number of antennas, see Figs. 7, 8.

Observe that extending the spacing leads to a narrowing of the mainlobe, whereas more antennas not only further decrease the mainlobe width but also increase the main-tosidelobe ratio. For example, in case of $d=2 \lambda_{c}, N=4$, the mainlobe width is approximately $15^{0}$ so that with such a linear array up to $180^{0} / 15^{0}=12$ spatially seperated users can be ideally served.

Note also that for $d=2 \lambda_{c}, N=2$ the array size is $8.76 \mathrm{~cm}$, which might be implementable on a terminal. In turn, for $d=2 \lambda_{c}, N=4$ the array size becomes $26.28 \mathrm{~cm}$, even this might be implementable - now on a laptop. 


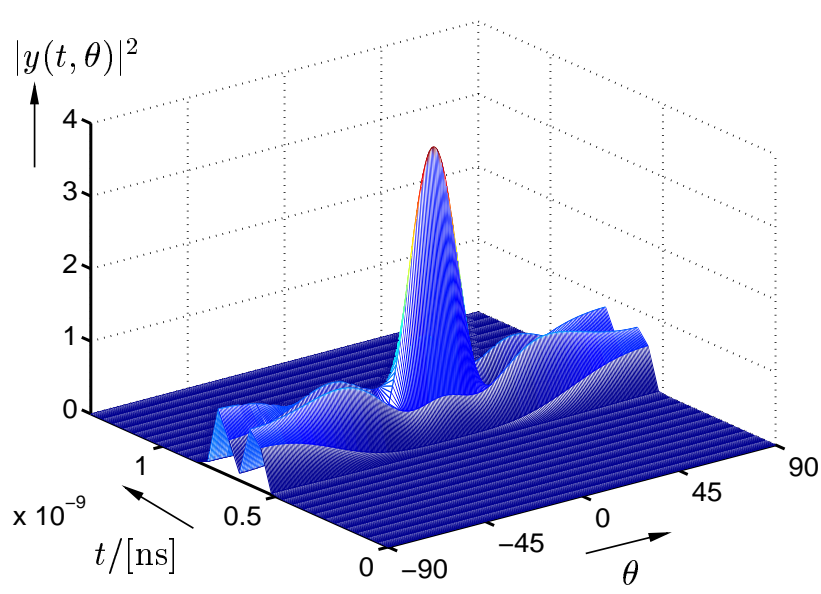

Fig. 5. Squared magnitude of broadband beamformer output for $d=\lambda_{c}, N=2$.

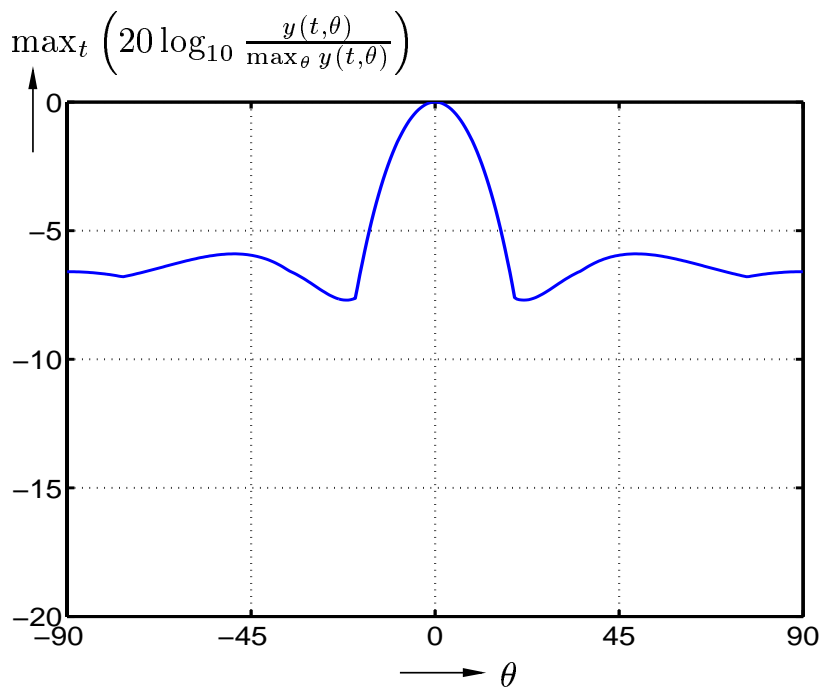

Fig. 6. Broadband normalized beampattern for $d=\lambda_{c}, N=2$ in logarithmic scale.

\section{Conclusion and future work}

In this contribution, we motivate the marriage of UWB and multi-antenna technique by focussing on beamforming. Beside the complementary classes of multi-antenna approaches, i.e. spatial multiplexing and space-time coding, there are numerous open issues in this new and highly multi-disciplinary field:

- adjustable precise analog true time delays (or even filters)

- direction of arrival, time delay estimation

- beamforming for UWB channels (model \& data)

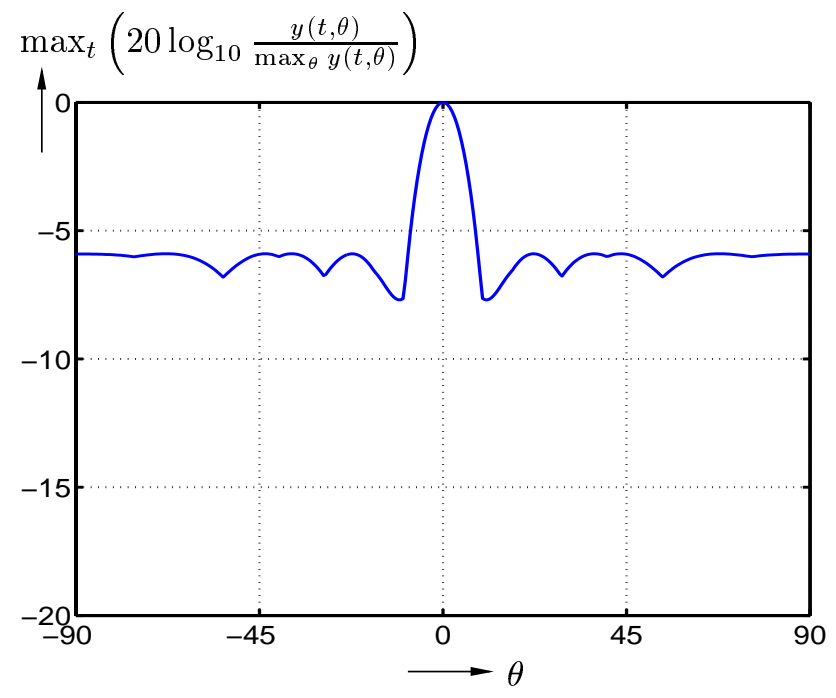

Fig. 7. Broadband normalized beampattern for $d=2 \lambda_{c}, N=2$ in logarithmic scale.

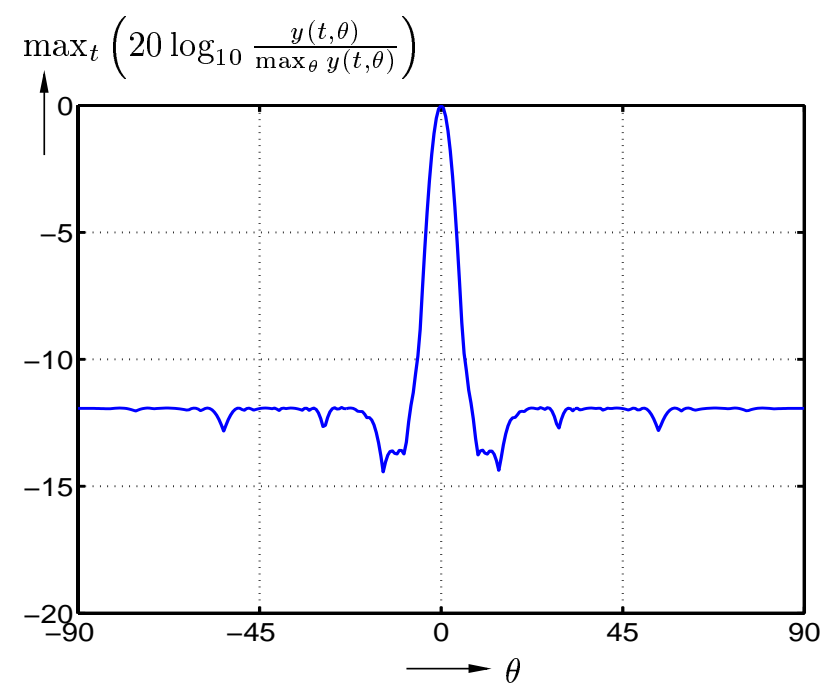

Fig. 8. Broadband normalized beampattern for $d=2 \lambda_{c}, N=4$ in logarithmic scale.

- exploiting multipath propagation than combating

- pulse shaping, modulation for UWB multi-antenna systems

- localisation, synchronisation with UWB multi-antenna systems

- UWB antenna arrays, where the frequency responses among the elements may differ, but should overlap

- broadband beamforming with low number of antennas

- transmit beamforming. 
Of further interest is how the channel capacity is affected by UWB transmission. First work towards this relevant topic can be found in Feng et al. (2004a, 2004b). It seems that the number of antennas has the same impact than the bandwidth, in fact, the equation known from narrowband MIMO systems for the ergodic channel capacity

$C_{e}=B N \log _{2}(1+\mathrm{SNR})$

also holds approximately true for UWB channels. Such result further motivate the merge of these two key technologies in wireless communications.

\section{References}

Cramer, J. M., Scholtz, R. A., and Win, M. Z.: On the analysis of UWB communcation channels, IEEE Military Communication Conference Proceedings (MILCOM), 2, 1191-1195, 1999.

Lee, H., Haan, H., Shin, Y., and Im, S.: Multipath Characteristics of Impulse Radio Channels, Proc. IEEE VTC, 15-18 May, Tokyo, Japan, 2487-2491, 2000.

Sibille, A. and Bories, S.: Space diversity for UWB communications, EPMCC, 22-25 April, Glasgow, UK, 2003.

www.whyless.org/public/wp5.htm.

Feng, Z., Kaiser, T., and Czylwik, A.: On the Evaluation of Channel Capacity of Multi-Antenna UWB Indoor Wireless Systems - Part I: Frequency Flat Case, IEEE Transactions on Communications, submitted, 2004a.

Feng, Z., Kaiser, T., and Czylwik, A.: On the Evaluation of Channel Capacity of Multi-Antenna UWB Indoor Wireless Systems Part II: Frequency Selective Case, IEEE Transactions on Communications, submitted, 2004b. 